\title{
Micromechanical Characterization of TiN Films on 9Cr18 Steels
}

\author{
Tai-Hua Zhang, Yong Huan
}

State Key Laboratory of Nonlinear Mechanics (LNM), Institute of Mechanics, Chinese

Academy of Sciences, Beijing 100080, China, Email: zhangth@lnm.imech.ac.cn

\section{Xiu-Lan Wang}

Beijing Research Institute of Aerospace Materials \& Technology, Beijing 100076, China

\begin{abstract}
TiN coatings $0.5 \mu \mathrm{m}$ thick were deposited by plasma arc deposition on $9 \mathrm{Cr} 18$ steels. Vickers indentations, depth-sensing indentations and nanoscratches were produced with different tips on a broad range of applied load. Mechanical behavior of hard and brittle thin coatings on soft and ductile metallic substrates was investigated and interpreted. It is demonstrated that depth-sensing indentation and nanoscratch tests can provide more information about the near-surface elastic-plastic deformation, friction and wear properties. Compared with $9 \mathrm{Cr} 18$ steels, solid lubricating effects for TiN and organism/TiN coatings are very marked.
\end{abstract}

Keywords: TiN coatings, microhardness, depth-sensing indentation, nanoscratch

\section{Introduction}

Titanium nitride (TiN) is an important engineering material, in particular in the fields of microelectronics, machinery, and cutting tool industry. Among the various physical vapor deposition (PVD) techniques for depositing TiN, plasma deposition is unique in providing better coating/substrate adhesion than most of the conventional PVD techniques. Therefore, a thorough understanding of the mechanical response of a coated component to applied loads is of both fundamental and technical importance to evaluate the surface hardness and friction for plasma treated materials.

In this study, we study the micromechanical properties of the TiN-coated stainless steel 9Crl8, using microhardness, depth-sensing indentation and nanoscratch methods.

\section{Experimental details}

\subsection{Coating preparation}

The substrates are stainless steel 9Cr 18 . They were previously polished to obtain surfaces with a very low roughness. Before deposition, the substrates had been successively cleaned in gasoline, acetone, ligroine in an ultrasonic bath. The steel samples were then winded in a rectangular rack for subsequent handling.

Deposition of TiN coatings was carried out by plasma arc method. After the steel samples were put into the vacuum chamber $\left(3 \times 10^{-3} \mathrm{~Pa}\right)$, the plasma beam bombarded the steel samples to clean them. A bias voltage was then added to the samples. Under certain condition the deposition rate was estimated which would give the TiN coating thickness of about $0.5 \mu \mathrm{m}$. Finally, a kind of organic coating was deposited on the 
TiN coating.

\subsection{Mechanical Characterization}

Surface observation was performed by an optical microscope(POLYVAR MET ${ }^{\star}$, Austria), coupled with a microhardness instrument (HV, load $200 \mathrm{~g}$, holding time $30 \mathrm{~s}$ ).

Depth-sensing indentation was performed using a Nano Indenter ${ }^{\star}$ XP made in MTS with a Berkovich diamond tip ${ }^{[1]}$. The surface hardness and modulus were measured from five indentations at the same depths $(1.0 \mu \mathrm{m})$ for $9 \mathrm{Cr} 18$ and TiN/9Crl 8 samples, respectively.

Scratch tests were performed with LFM(Lateral Force Measurement) option of the Nano Indenter ${ }^{\otimes P}$. Berkovich tip was used with face forward. Normal load measurements were carried out by the linearly increasing load from $20 \mu \mathrm{N}$ to $40 \mathrm{mN}, 100 \mathrm{mN}, 300 \mathrm{mN}$ at a scratch speed of $10 \mu \mathrm{m} / \mathrm{s}$ (length $500 \mu \mathrm{m}$ ).

\section{Results and discussion}

\subsection{Microhardness}

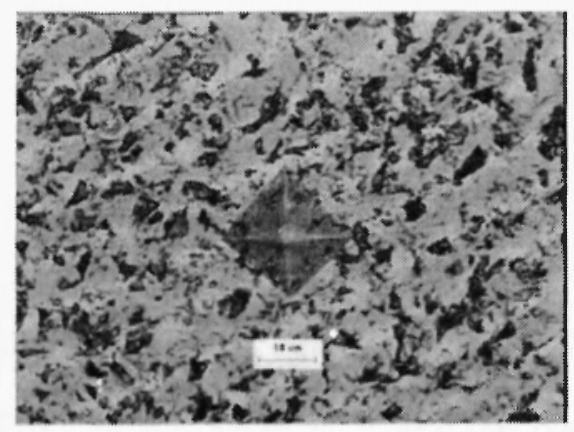

Fig. 1. Optical micrograph of steel $9 \mathrm{Cr} 18$

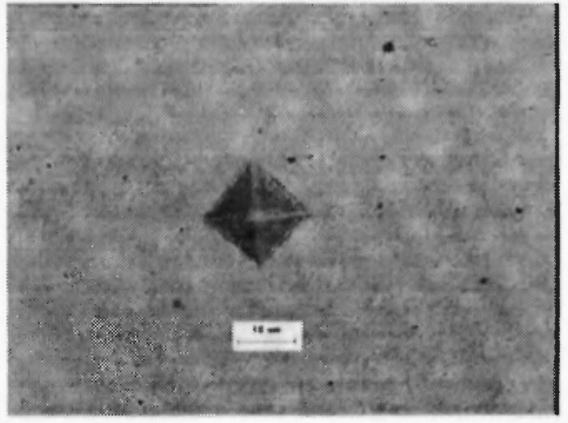

Fig. 2. Optical micrograph of $\mathrm{TiN} / 9 \mathrm{Cr} 18$
In the first series of experiments, we evaluated the mechanical properties of $9 \mathrm{Cr} 18$ steel and $\mathrm{TiN} / 9 \mathrm{Cr} 18$ surfaces using the microhardness indentation technique. Typical indentation micrographs are shown in Fig. 1 and Fig. 2. The indentation area for $9 \mathrm{Cr} 18$ steel is well marked. The values for five indentations are $H V_{0.2}=682.88 \pm 24.77$. The indentation edge of TiN/9Cr18 is so blurry that we could not measure exactly the indentation area. So, exact values were not given. It is also shown that TiN coatings exhibit well elastic deformation behavior.

\subsection{Depth-sensing indentation}

The load, hardness and modulusdisplacement curves for $9 \mathrm{Cr} 18$ and $\mathrm{TiN} / 9 \mathrm{Cr} 18$ are shown in Fig. 3 and Fig. 4, respectively. For TiN/9Cr18 in Fig. 4, the hardness and modulus values for TiN coatings are displayed between $50 \mathrm{~nm}$ and $100 \mathrm{~nm}$. With displacement into surface increasing, hardness and modulus represent composite values, consisting of a contribution from the very thin modified surface layer $\mathrm{TiN}$ and the steel $9 \mathrm{Cr} 18$ bulk. When the depths are $1000 \mathrm{~nm}$, the contribution of the underlying softer $9 \mathrm{Cr} 18$ became more pronounced. The Hardness and Modulus values for $9 \mathrm{Cr} 18$ appeared to be far lower than TiN. It make against improving adhesion of coatings. So, the modulus and hardness of substrates should approach to TiN when choosing substrate materials.

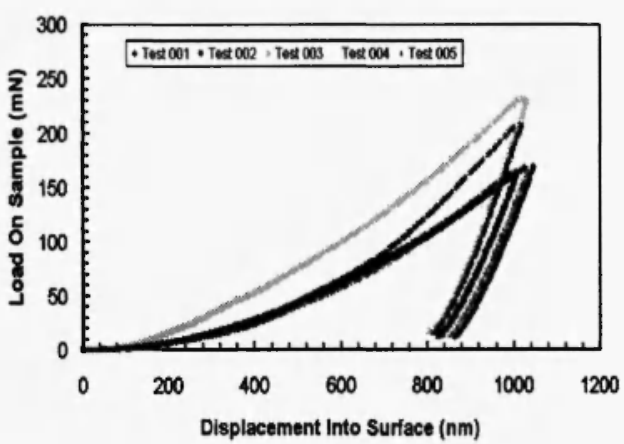



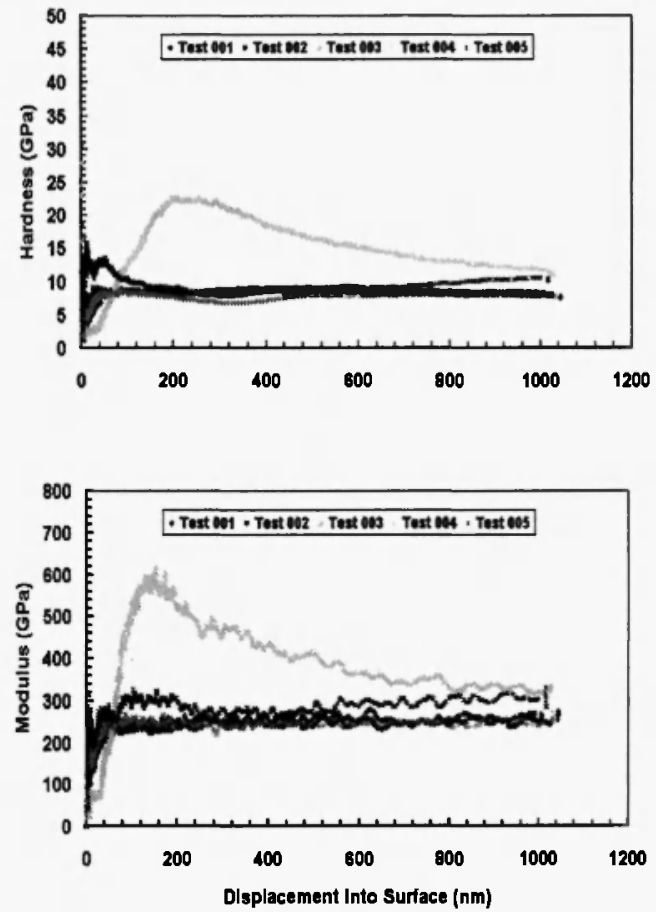

Fig. 3. Steel $9 \mathrm{Cr} 18$
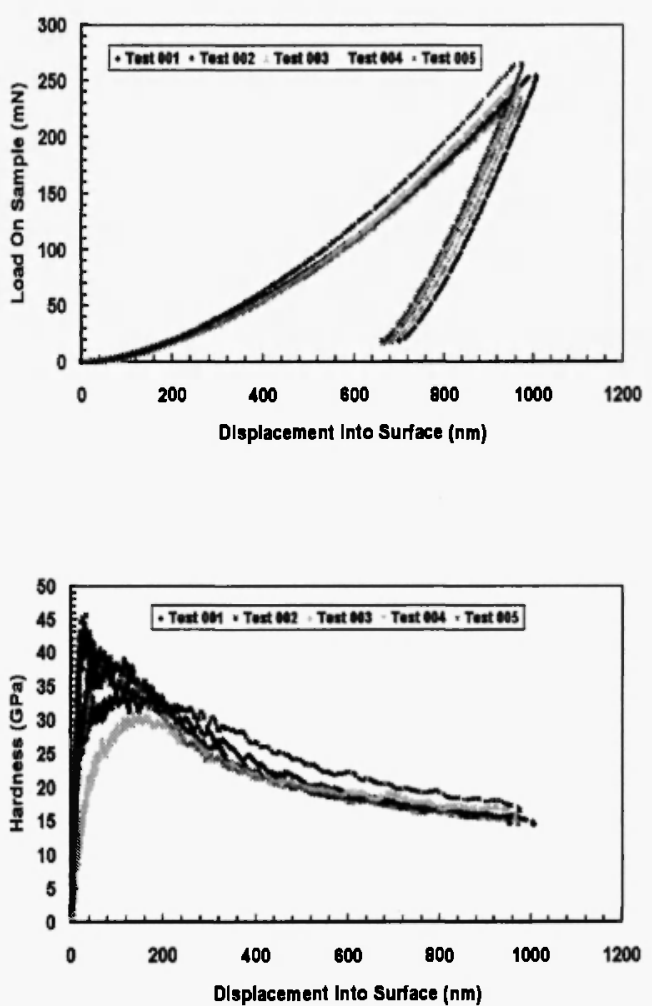

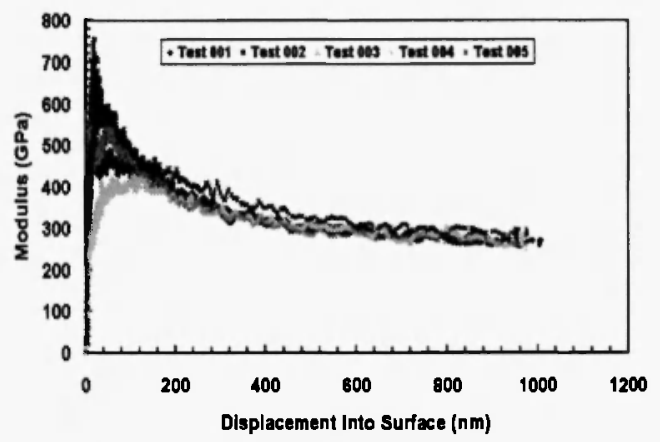

Fig. 4. TiN/9Cr18

\subsection{Nanoscratch}

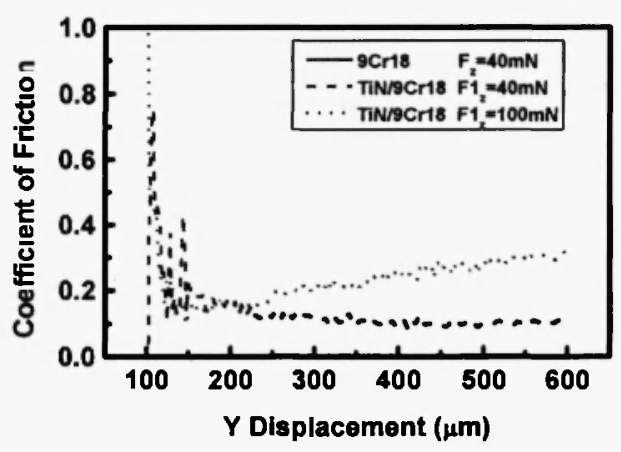

Fig. 5. TiN coating

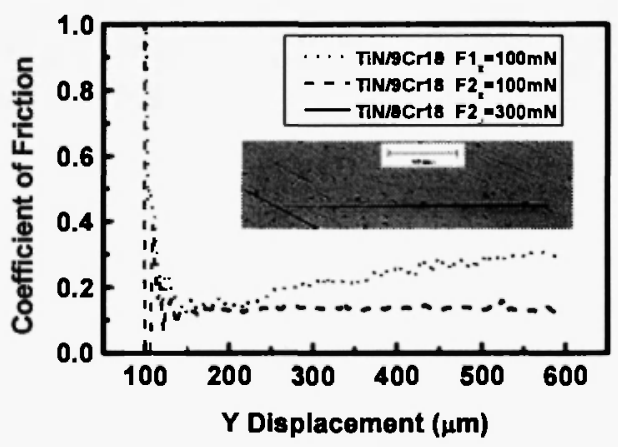

Fig. 6. Organism/TiN composite coating

In comparison with the steel $9 \mathrm{Cr} 18$ in Fig. 5, the friction coefficient at Max. normal load $40 \mathrm{mN}$ decreases from 0.4 for the uncoated steel 
to 0.1 for $\mathrm{TiN} / 9 \mathrm{Cr} 18$. The friction coefficient increasing for $\mathrm{TiN}$ coating at $100 \mathrm{mN}$ clearly represents the tip scratching from $\mathrm{TiN}$ coating to steel substrate.

In comparison with the TiN coating in Fig. 6 , no significant change is detected for the friction coefficient at $100 \mathrm{mN}$ for the organism/TiN coating. However, the tip at $300 \mathrm{mN}$ scratched into the steel. Except for scratch tests for $F l_{z}=40 m N$ in Fig. 5 and $F 2 z=100 m N$ in Fig. 6, pronounced damage tracks can be seen by the optical microscope, such as the micrograph for $F 2,=300 \mathrm{mN}$ test.

\section{Conclusion}

We have studied the micro-mechanical properties of the solid lubricating coatings. The main conclusions may be summarized as follows:
1) The depth-sensing indentation and scratch tests can provide more information about the near-surface elastic-plastic deformation, friction and wear properties.

2) When choosing materials, modulus and hardness for substrates and coatings should be matching.

3) Compared with the steel $9 \mathrm{Cr} 18$, solid lubricating effects for TiN and organism/TiN coatings are very marked.

\section{Acknowledgments}

This research was supported by the National Natural Science Foundation of China under Grant No.10102021 and No. 10172086.

\section{References}

[1] www.mts.com/nano 